\title{
40 \\ Genetic neuro-fuzzy architectures for advanced intelligent systems
}

\author{
Sung-Bae Cho
}

Dept. of Computer Science, Yonsei University, 134 Shinchon-dong, Sudaemoon-ku, Seoul 120-749, Korea (sbcho@csai.yonsei.ac.kr)

ATR Human Information Processing Research Laboratories, 2-2 Hikaridai, Seika-cho, Soraku-gun, Kyoto 619-02, Japan

\begin{abstract}
This paper presents a framework for developing intelligent systems based on several softcomputing techniques such as fuzzy logic, neural networks and genetic algorithm. The neural networks provide the system with a baseline structure, the fuzzy logic gives a possibility to utilize top-down knowledge from designer, and the genetic algorithm determines several system parameters with the process of bottom-up development. As a manifestation, we propose an efficient fuzzy neural system which consists of modular neural networks combined by the fuzzy integral in which genetic algorithm determines the fuzzy density values.
\end{abstract}

\section{Keywords}

Intelligent systems, softcomputing, neural networks, fuzzy logic, genetic algorithm

\section{INTRODUCTION}

Fuzzy logic, neural networks and genetic algorithm are three technologies that have been proposed for achieving some aspect of intelligent systems. Their differences, however, have prompted us to try combining them to produce more powerful systems (Cho \& Kim, 1992; Cho, 1994a; Cho, 1994b; Cho \& Kim, 1995). In this paper we introduce a framework for combining three of them, and present an efficient classification system as an implementation. 
- Fuzzy logic : Fuzzy logic is characterized as extension of binary crisp logic. Each fuzzy rule has an antecedent part containing several preconditions, and a consequent part which prescribes the value. The fuzzy set is a class in which transition from membership to non-membership is gradual rather than abrupt. Crisp sets allow only full membership or no membership at all, whereas fuzzy sets allow partial membership. In other words, an element may partially belong to a set. Traditionally, fuzzy logic uses minimum and maximum operators.

- Neural networks : Neural networks, a model of the brain, artificially connects many nonlinear neuron model and processes information in a parallel distributed manner. The neural network has many characteristics such as nonlinear mapping, parallel processing, learning, and self-organization. It is applied to pattern recognition, control and so on. The neural network which consists of three layers (input/output layers and one hidden layer) are able to express any functions while using enough hidden units.

- Genetic algorithm : Genetic algorithm is one of search methods based on the mechanics of natural selection and natural genetics. It is not a gradient search technique. It combines survival of the fittest among string structures with a structured but randomized information exchange to form a search algorithm with some of the innovative flair of human problem solving. An occasional new part is attempted for a good measure. While randomized, genetic algorithms do not perform simple random walk. They efficiently exploit historical information to speculate on new search points with expected improved performance. The two operators used frequently are crossover and mutation.

As described above, each method has it's own merits and demerits. To produce more powerful system, several integration and synthesis techniques of them have been proposed. This paper presents a framework for high-performance classification system based on them. The neural networks can be used as a baseline system, because they are well recognized as a powerful input-output mapper. One of the weakest points, however, is that human operators cannot easily provide with any knowledge about the problem at hand. In this case, the fuzzy logic is useful.

To give an idea of how such hybrid technique yields better classification system, we developed a neuro-fuzzy system that considers the difference of performance of each network in combining the networks. It is based on the notion of fuzzy logic, especially the fuzzy integral. This method combines the outputs of separate networks with importance of each network, which is assigned by genetic algorithm.

\section{MULTIPLE NEURAL NETWORKS}

Suppose a two-layer neural network classifier with $T$ neurons in the input layer, $H$ neurons in the hidden layer, and $c$ neurons in the output layer. Here, $T$ is the number of features, $c$ is the number of classes, and $H$ is an appropriately selected number. The network is fully connected between adjacent layers. The operation of this network can be thought of as a nonlinear decision-making process: Given an unknown input $X=\left(x_{1}, x_{2}, \ldots, x_{T}\right)$ 


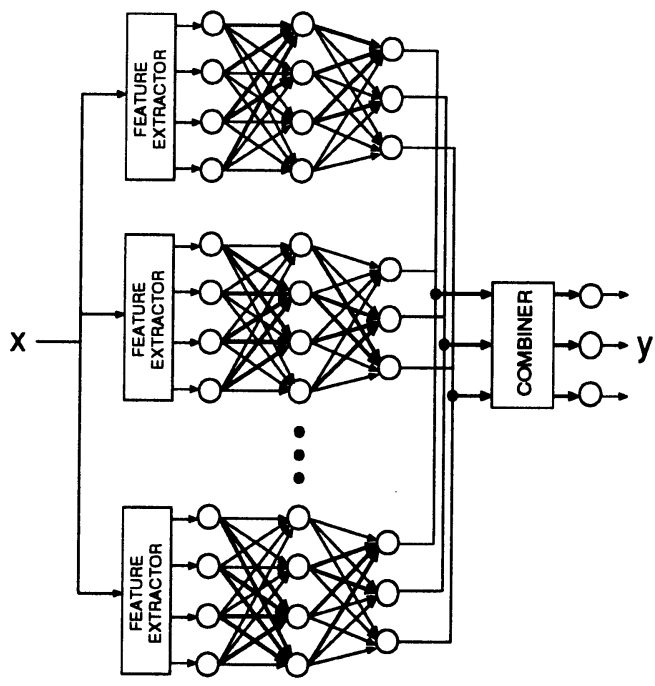

Figure 1 Multiple neural networks combined by fusion method.

and the class set $\Omega=\left\{\omega_{1}, \omega_{2}, \ldots, \omega_{c}\right\}$, each output neuron produces $y_{i}$ of belonging to this class by

$P\left(\omega_{i} \mid X\right) \approx f\left\{\sum_{k=1}^{H} w_{i k}^{o m} f\left(\sum_{j=1}^{T} w_{k j}^{m i} x_{j}\right)\right\}$

where $w_{k j}^{m i}$ is a weight between the $j$ th input neuron and the $k$ th hidden neuron, $w_{i k}^{o m}$ is a weight from the $k$ th hidden neuron to the $i$ th class output, and $f$ is a sigmoid function such as $f(x)=1 /\left(1+e^{-x}\right)$. The neuron having the maximum value is selected as the corresponding class.

The network presented above trains on a set of example patterns and discovers relationships that distinguish the patterns. A network of a finite size, however, does not often load a particular mapping completely or it generalizes poorly. Increasing the size and number of hidden layers most often does not lead to any improvements. Furthermore, in complex problems such as character recognition, both the number of available features and the number of classes are large. The features are neither statistically independent nor unimodally distributed. The basic idea of the multiple network scheme is to develop $n$ independently trained neural networks with relevant features, and to classify a given input pattern by utilizing combination methods to decide the collective classification (Hansen \& Salamon, 1990) (Figure 1). Then it naturally raises the question of obtaining a consensus on the results of each individual network or expert. 


\section{FUZZY LOGIC BASED METHOD}

The fuzzy integral is a nonlinear functional that is defined with respect to a fuzzy measure, especially $g_{\lambda}$-fuzzy measure introduced by Sugeno (Sugeno, 1977). The ability of the fuzzy integral to combine the results of multiple sources of information has been established in the previous work (Tahani \& Keller, 1990). Using the notion of fuzzy measures, Sugeno developed the concept of the fuzzy integral, which is a nonlinear functional that is defined with respect to a fuzzy measure, especially $g_{\lambda}$-fuzzy measure (Sugeno, 1977; Tahani \& Keller, 1990).

Definition: Let $X$ be a finite set, and $h: X \rightarrow[0,1]$ be a fuzzy subset of $X$. The fuzzy integral over $X$ of the function $h$ with respect to a fuzzy measure $g$ is defined by

$$
\begin{aligned}
h(x) \circ g(\cdot) & =\max _{E \subseteq X}\left[\min \left(\min _{x \in E} h(x), g(E)\right)\right] \\
& =\sup _{\alpha \in[0,1]}\left[\min \left(\alpha, g\left(h_{\alpha}\right)\right)\right]
\end{aligned}
$$

where $h_{\alpha}$ is the $\alpha$ level set of $h$,

$h_{\alpha}=\{x \mid h(x) \geq \alpha\}$.

To get some intuition for the fuzzy integral we consider the following interpretation. $h(y)$ measures the degree to which the concept $h$ is satisfied by $y$. The term $\min _{y \in E} h(y)$ measures the degree to which the concept $h$ is satisfied by all the elements in $E$. Moreover, the value $g(E)$ is a measure of the degree to which the subset of objects $E$ satisfies the concept measured by $g$. Then, the value obtained from comparing these two quantities in terms of the min operator indicates the degree to which $E$ satisfies both the criteria of the measure $g$ and $\min _{y \in E} h(y)$. Finally, the max operation takes the biggest of these terms. One can interpret the fuzzy integral as finding the maximal grade of agreement between the objective evidence and expectation.

Let $\Omega=\left\{\omega_{1}, \omega_{2}, \ldots, \omega_{c}\right\}$ be a set of classes of interest. Note that each $\omega_{i}$ may, in fact, be a set of classes by itself. Let $Y=\left\{y_{1}, y_{2}, \ldots, y_{n}\right\}$ be a set of neural networks, and $A$ be the object under consideration for recognition. Let $h_{k}: Y \rightarrow[0,1]$ be the partial evaluation of the object $A$ for class $\omega_{k}$, that is, $h_{k}\left(y_{i}\right)$ is an indication of how certain we are in the classification of object $A$ to be in class $\omega_{k}$ using the network $y_{i}$, where a 1 indicates absolute certainty that the object $A$ is really in class $\omega_{k}$ and 0 implies absolute certainty that the object $A$ is not in $\omega_{k}$.

\section{GENETIC ALGORITHM BASED METHOD}

Evolution is a remarkable problem-solving machine (Srinivas \& Patnaik, 1994). First proposed by John Holland in 1975, GAs as one of computational implementations are an attractive class of computational models that mimic natural evolution to solve problems in a wide variety of domains. A genetic algorithm emulates biological evolutionary theories to solve optimization problems.

The basis of a GA is that a population of problem solutions is maintained in the form 
of chromosomes, which are strings encoding problem solutions. Strings can be binary or have many possible alternatives (genes) at each position. The strings are converted into problem solutions, which are then evaluated according to an objective scoring function. Often it is not possible to exhaustively test all aspects of a solution, and noise may be present on the objective function, so the assigned fitness is an estimate of the true fitness of a chromosome. It is important that this is a good estimate, otherwise the selective pressure that favors truly high scoring chromosomes can be lost in the noise caused by poor fitness estimates.

Following fitness evaluation, a new population of chromosomes is generated by applying a set of genetic operators to the original population. These are basically random copying and altering of individuals from the original population with the probability of copying of any individual from one generation to the next being proportional to its fitness. During the copying process two operations may be performed-a gene may be erroneously copied (mutation), or a new individual may be formed by combining segments from two chromosomes by copying one chromosome up to a specific location on the chromosome, then copying a different chromosome (crossover).

In computing terms, a genetic algorithm maps a problem onto a set of strings, each string representing a potential solution. In our problem, a string must encode $n \times c$ parameters, thereby optimal combination coefficients for combining neural networks can be obtained. The GA then manipulates the most promising strings in its search for improved solutions.

\section{GENETIC FUZZY HYBRID METHOD}

In general, fuzzy logic gives a possibility to utilize top-down knowledge from designer. On the other hand, genetic algorithm is a powerful tool for structure optimization of the fuzzy logic and the neural networks which provide an evaluation functions for the genetic algorithm. Figure 2 shows a schematic diagram of the framework based on the hybridization of them.

To give an idea of how such hybrid technique yields better system, the hybrid method utilizes the fuzzy integral to combine the outputs of separate networks with importance of each network, which is assigned by genetic algorithm. In the following, $\hat{g}_{\lambda}(A)$ and $\hat{g}_{i}$ denote the human-provided values, and $g_{\lambda}(A)$ and $g_{i}$ denote the identified values.

In this method, chromosomes encode the fuzzy density values $g_{i}^{j}$ by a vector $C_{j}=$ $\left(g_{1}^{j}, g_{2}^{j}, \ldots, g_{k}^{j} ; \lambda_{j}\right)$. The fitness function $f\left(C_{j}\right)$ for chromosome $C_{j}$ is the sum of the differences between human-provided fuzzy measure value $\hat{g}_{\lambda}(A)$ and fuzzy measure value obtained by $g_{i}^{j}$ and $\lambda_{j}$.

$f\left(C_{j}\right)=\sum_{A \in B(X)}\left|\hat{g}_{\lambda}(A)-\frac{1}{\lambda_{j}}\left[\prod_{x_{i} \in A}\left(1+\lambda_{j} g_{i}^{j}\right)-1\right]\right|$

With these the genetic operators yield an optimal set of parameters to combine neural networks. 


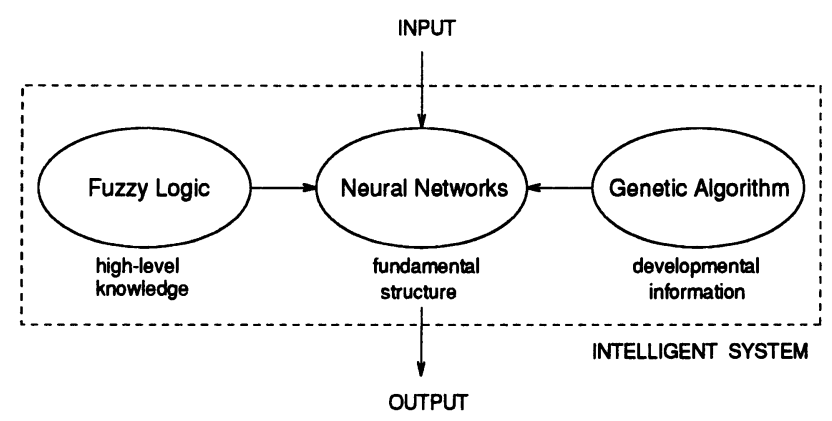

Figure 2 Schematic diagram of the hybrid intelligent system.

\section{EXPERIMENTAL RESULTS}

In the experiments, we have used the handwritten numeral database of Concordia University of Canada, which consists of 6000 unconstrained numerals originally collected from dead letter envelopes by the U.S. Postal Services at different locations in the U.S. The numerals of this database were digitized in bilevel on a $64 \times 224$ grid of $0.153 \mathrm{~mm}$ square elements, giving a resolution of approximately 166 PPI (Suen et al., 1990). Among the data, 4000 numerals were used for training and 2000 numerals for testing.

To evaluate the performance of the multiple NN classifier, we have implemented three different networks, each of which is a two-layer neural network using different features. $\mathrm{NN}_{1}, \mathrm{NN}_{2}$ and $\mathrm{NN}_{3}$ have used the normalized image, Kirsch features (Pratt, 1978), and the sequence of contour features, respectively. In this fashion each network makes the decision through its own criterion. For the fuzzy logic based method, we assigned the fuzzy densities $g^{i}$, the degree of importance of each network, based on how good these networks performed on validation data. Figure 3 shows the fitness changes as generation goes with respect to the two different mutation rates. As the figure dictates, it is not appropriate to choose the mutation rate as larger than $1 \%$, and we used the genetic algorithm with $1 \%$ mutation rate.

Table 1 shows the recognition rates with respect to the three different networks and their combinations by utilizing consensus methods like majority voting and average, as well as the fuzzy integral, genetic algorithm and the hybrid of them. $\mathrm{NN}_{\text {all }}$ here means the network trained with all the available features. The reliability in the table is computed as the following equation:

$$
\text { Reliability }=\frac{\text { Correct Recognition Rate }}{\text { Correct Recognition Rate }+ \text { Substitution Error Rate }} \text {, }
$$

where the Substitution Error Rate is the portion of patterns which are classified incorrectly by the method. As can be seen, any method of combining multiple NN produces better results than individual networks, and the overall classification rates for the soft- 


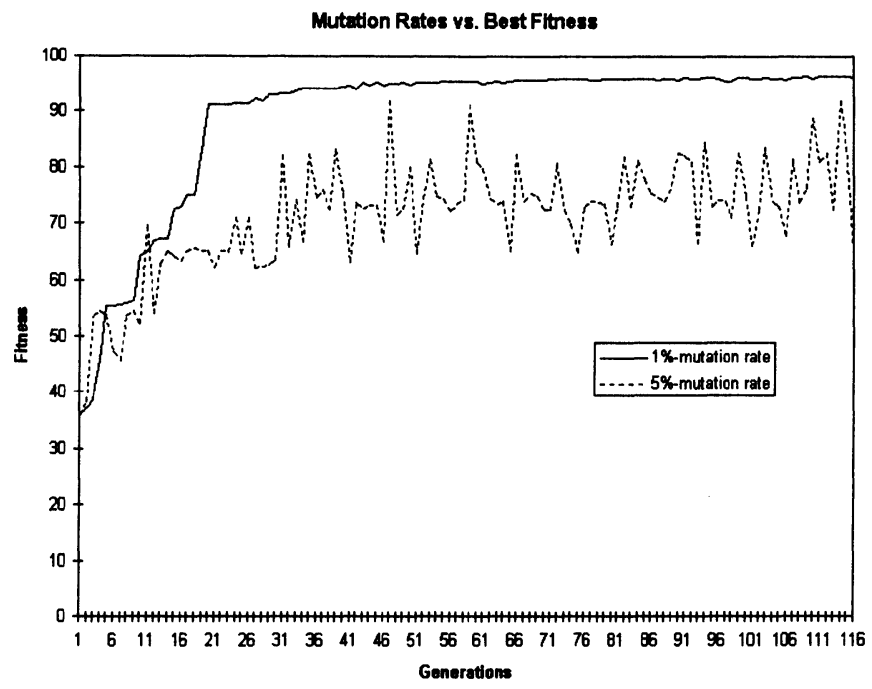

Figure 3 Fitness changes with respect to different mutation rates.

Table 1 The result of recognition rates (\%).

\begin{tabular}{ccccc}
\hline Methods & Recognized & Substituted & Rejected & Reliability \\
\hline $\mathrm{NN}_{1}$ & 89.05 & 7.00 & 3.95 & 92.71 \\
$\mathrm{NN}_{2}$ & 95.40 & 3.75 & 0.85 & 96.22 \\
$\mathrm{NN}_{3}$ & 93.95 & 4.10 & 1.95 & 95.82 \\
\hline $\mathrm{NN}_{\text {all }}$ & 95.85 & 4.15 & 0.00 & 95.85 \\
\hline Voting & 96.70 & 3.05 & 0.25 & 96.94 \\
Average & 97.15 & 2.35 & 0.50 & 97.64 \\
\hline Fuzzy & 97.35 & 2.30 & 0.35 & 97.69 \\
Genetic & 97.90 & 2.10 & 0.00 & 97.90 \\
Hybrid & 98.05 & 1.95 & 0.00 & 98.05 \\
\hline
\end{tabular}

computing techniques are higher than those for other consensus methods. Although the network learned the training set almost perfectly in all three cases, the performances on the test sets are quite different. Furthermore, we can see that the performance did not improve by training a large network with considering all the features used by each network. This is a strong evidence that multiple neural networks might produce better result than conventional single network approach. 


\section{CONCLUDING REMARKS}

This paper has presented several softcomputing techniques to produce an improved performance on real-world classification problem, especially handwritten numeral recognition. One of the important advantages of the methods is that not only is the classification results combined but that the relative importance of the different networks is also considered. The experimental results for classifying a large set of handwritten numerals show that it improves the generalization capability significantly. This indicates that even these straightforward, computationally tractable approach can significantly enhance pattern recognition.

\section{REFERENCES}

Cho, S.-B. \& Kim, J.H. (1992) Two design strategies of neural network for complex classification problems. Proc. 2nd Int. Conf. Fuzzy Logic \& Neural Net. 759-762.

Cho, S.-B. (1994a) Cooperation of modularized neural networks by fuzzy integral with OWA operators. 3rd Int. Conf. Fuzzy Logic, Neural Nets, and Soft Computing 95-96.

Cho, S.-B. (1994b) Neural network ensemble aggregated by fuzzy logic. IEEE/Nagoya University World Wisemen/women Workshop on Fuzzy Logic and Neural Networks/Genetic Algorithms 46-52.

Cho, S.-B. \& Kim, J.H. (1995) Combining multiple neural networks by fuzzy integral for robust classification. IEEE Trans. Syst. Man. Cyber. 25 380-384.

Hansen, L.K. \& Salamon, P. (1990) Neural network ensembles. IEEE Trans. Patt. Anal. Mach. Inte. 12 993-1001.

Pratt, W.K. (1978) Digital Image Processing. New York: Wiley.

Srinivas, M. \& Patnaik, L.M. (1994) Genetic algorithms: a survey. IEEE Computer, 17-26, June.

Suen, C.Y., Nadal, C., Mai, T., Legault, R. \& Lam, L. (1990) Recognition of handwritten numerals based on the concept of multiple experts. In Proc. 1st Int. Workshop Frontiers in Handwriting Recognition, Montreal, Canada, 131-144.

Sugeno, M. (1977) Fuzzy measures and fuzzy integrals: A survey. Fuzzy Automata Dec. Proc. 89-102 (Amsterdam: North Holland).

Tahani, H. \& Keller, J.M. (1990) Information fusion in computer vision using the fuzzy integral. IEEE Trans. Syst. Man. Cyber. 20 733-741.

SUNG-BAE CHO received the B.S. degree in computer science from Yonsei University, Korea and the M.S. and Ph.D. degrees in computer science from KAIST (Korea Advanced Institute of Science and Technology), Korea. He worked as a Research Staff at the Center for Artificial Intelligence Research at KAIST from 1991 to 1993 . He was an Invited Researcher of Human Information Processing Research Laboratories at ATR (Advanced Telecommunications Research) Institute, Kyoto, Japan from 1993 to 1995 . Since 1995, he has been an Assistant Professor in the Department of Computer Science, Yonsei University, Seoul, Korea. 